\title{
Toward a Global Understanding of the Homotopy Groups of Spheres
}

\author{
Mark E. Mahowald \\ and \\ Douglas C. Ravenel
}

\begin{abstract}
In this paper we will describe a point of view that has emerged as a result of research on the homotopy groups of spheres in the last decade. This philosophy is difficult to translate into theorems or even into precise conjectures, and it is certainly not apparent in the formal literature on the subject. With the exception of Theorem 10, we will not present any proofs or announcements of new results here. Rather we will collect numerous old results and current ideas and arrange them into what we hope is a suggestive picture.
\end{abstract}

\section{General facts about homotopy groups}

For the last 50 years one of the basic problems in algebraic topology has been the determination of the homotopy groups of spheres $\pi_{n+k}\left(S^{n}\right)$, i.e. the classification of continuous maps

$$
S^{n+k} \rightarrow S^{n}
$$

up to continuous deformation. The simplicity of the spaces involved lends intuitive appeal to the problem, but experience has shown that it is as hard as any in mathematics. There have been several major computational breakthroughs in the subject, namely the EHP sequence (to be described in Section 7 below), and the spectral sequences of Serre, Adams and Novikov. Each of these had lead to a large amount of new information but has also increased our appreciation of the difficulty of the problem. Much of this material is dealt with in greater depth and with numerous references in [R1].

We begin by recalling some of the basic facts about the problem. All of these groups are abelian and finitely generated. The groups $\pi_{n+k}\left(S^{n}\right)$ are known to vanish when $k<0$ and when $n=1$ and $k>0$. The group $\pi_{n}\left(S^{n}\right)$ is isomorphic to the integers $Z$. These were all proved by Hurewicz around 1935. Their finite computability was established by E.H. Brown in 1959.

The following finiteness result was proved by Serre.

Theorem 1. [S]. The groups $\pi_{n+k}\left(S^{n}\right)$ for $k>0$ are all finite with the exception of $\pi_{4 n-1}\left(S^{2 n}\right)$, which is the direct sum of $Z$ and a finite abelian group. Hence for $n$ odd the standard map

$$
S^{n} \rightarrow K(Z, n)
$$


induces an isomorphism in homotopy mod torsion, where $K(Z, n)$ denotes the integer Eilenberg-MacLane space.

Shortly after the groups were defined Freudenthal proved that $\pi_{n+k}\left(S^{n}\right)$ is independent of $n$ when $n>k+1$. These groups are said to be stable and the value of $\pi_{n+k}\left(S^{n}\right)$ for large $n$ is called the stable $k$-stem and denoted by $\pi_{k}^{S}\left(S^{0}\right)$ or simply $\pi_{k}^{S}$.

The groups $\pi_{n+k}\left(S^{n}\right)$ with $n \leq k+1$ are called unstable. We have more machinery for computing stable groups than unstable ones. One of the major themes in the subject has been the attempt to bring the more advanced technology of stable homotopy to bear on the problems of unstable homotopy theory. Two early examples of this are [A1] and [M1].

Even the stable groups are quite mysterious. The following table gives their values for small $k$.

$\begin{array}{llll}k & \pi_{k}^{S} & k & \pi_{k}^{S} \\ 0 & Z & 7 & Z /(240) \\ 1 & Z /(2) & 8 & (Z /(2))^{2} \\ 2 & Z /(2) & 9 & (Z /(2))^{3} \\ 3 & Z /(24) & 10 & Z /(6) \\ 4 & 0 & 11 & Z /(504) \\ 5 & 0 & 12 & 0 \\ 6 & Z /(2) & 13 & Z /(3)\end{array}$

As the reader can see, these groups do not fall into any obvious pattern. However, there is a certain overall structure which we shall describe presently.

These groups form a graded ring under smash product of maps between spheres in the following way.

Given $\alpha \in \pi_{j}^{S}$ and $\beta \in \pi_{k}^{S}$, choose maps

$$
\begin{aligned}
& f: S^{m+j} \rightarrow S^{m} \quad \text { and } \\
& g: S^{n+k} \rightarrow S^{n}
\end{aligned}
$$

for $m$ and $n$ sufficiently large. Then the smash product $f \wedge g$ represents the class $\alpha \beta$. It can be shown that this product is commutative up to the usual sign.

There is also a product defined in terms of composition of maps. As long as everything is in the stable range, the composition and smash products agree. However, the composition product fails to commute (even up to sign) in general. For example, if

$$
f: S^{3} \rightarrow S^{2}
$$

is the Hopf map then one has

$$
f \cdot 4=2 \cdot f
$$

where "2" denotes the degree 2 map on $S^{2}$ and " 4 " denotes the degree 2 map on $S^{4}$.

This noncommutativity is very important in unstable homotopy theory.

In $[\mathrm{N}]$ Nishida proved that every positive dimensional element in this ring is nilpotent, i.e. for each $\alpha \in \pi_{k}^{S}$ with $k>0$, there is an exponent $m$ such that $\alpha^{m}=0$. A vast generalization of this nilpotence result conjectured in [R2] has been proved recently by Devinatz, Hopkins and Smith and will be commented on below (see Theorem 6). Nishida's 
Theorem indicates that the multiplicative structure of this ring is of very limited use. One should not attempt to describe it in terms of generators and relations.

\section{Periodic families}

A construction which has proved quite useful is the following. Suppose we have a finite complex $Y$ and a map

$$
v: \Sigma^{d} Y \rightarrow Y
$$

such that all iterates of the form

$$
v^{i}=v \cdot \Sigma^{d} v \cdots \Sigma^{(i-1) d} v: \Sigma^{i d} Y \rightarrow Y
$$

are essential. It is convenient for technical reasons to require that the complex $Y$ and the self-map $v$ are both double suspensions. A map of this sort all of whose iterates are essential (i.e. not homotopic to the constant map) is said to be periodic.

The set of homotopy classes of maps from $\Sigma^{k} Y$ to a space $X$ is an abelian group, which we will denote by $\pi_{k}(X ; Y)$. (We reserve the right to change the index $k$ when convenient.) Given an element $\alpha \in \pi_{k}(X ; Y)$ represented by a map

$$
f: \Sigma^{k} Y \rightarrow X
$$

we can define $v^{i} \alpha \in \pi_{k+i d}(X ; Y)$ to be the class represented by the composite $f \cdot \Sigma^{k} v^{i}$.

This makes the graded group $\pi_{*}(X ; Y)$ a module over the ring $Z[v]$. If the finite complex $Y$ is such that the identity map has order $q$ in the group $[Y, Y]$ then $\pi_{*}(X ; Y)$ is a module over the ring $Z /(q)[v]$. In any case one can tensor with $Z\left[v, v^{-1}\right]$ and ask the following question.

What is $v^{-1} \pi_{*}(X ; Y)$ ?

An element in $\pi_{*}(X ; Y)$ has a nontrivial image in this group iff it is not annihilated by any power of $v$. Such elements are said to be $v$-periodic. Elements which are annihilated by some power of $v$ are said to be $v$-torsion. In Section 5 we will introduce $B P$-theory and explain how it provides us with some powerful methods for computing or at least estimating this group. A more delicate question is that of the image of $\pi_{*}(X ; Y)$ in it. An answer to this question will lead to some useful information about $\pi_{*}(X)$ itself.

A $v$-periodic family in $\pi_{*}(X ; Y)$ is a set of elements of the form

$$
\left\{x, v x, v^{2} x, \cdots\right\}
$$

such that $v^{i} x$ is nontrivial for all $i>0$.

The finiteness theorem of Serre mentioned above can be regarded as a result of this type. Let $X=S^{n}, Y=S^{1}$ and let $v$ be the degree $p$ map for $p$ a prime. Then we are asking for the structure of $p^{-1} \pi_{*}\left(S^{n}\right)$. If we localize everything in sight at the prime $p$ we get rid of torsion prime to $p$, so the image of $\pi_{*}\left(S^{n}\right)$ is its torsion free quotient. For $n$ odd Serre's theorem says that the standard map from $S^{n}$ to $K(Z, n)$ induces a homotopy equivalence modulo torsion, i.e. the map is a rational homotopy equivalence.

Notice that Nishida's theorem tells us that if we take our finite complex $Y$ to be a sphere of any dimension then the degree $p$ map is essentially the only possible choice for the self map $v$. Any map coming from a positive stem would have to be nilpotent and therefore would not be suitable. The result of Devinatz-Hopkins-Smith (see Theorem 6 below), which generalizes Nishida's theorem tells us that the same is true whenever 
$H_{*}(Y)$ is torsion free. It turns out that using any such $Y$ will give us essentially the same information about $\pi_{*}(X)$, namely its torsion free quotient.

\section{The stable image of $J$ as a periodic family}

In view of the remarks in the previous paragraph we should consider a finite complex $Y$ with some torsion in its homology. Let $M(p)$ denote a mod $(p)$ Moore space. We do not care about the dimension of its bottom cell, as long as it is not too small. We will use the same notation for the mod $(p)$ Moore spectrum with its bottom cell in dimension 0 . This is now our finite complex $Y$. The group $\pi_{*}(X ; Y)$ is called the $\bmod (p)$ homotopy of $X$. The self map $v$ is provided by the following result of Adams.

Lemma 2. [A2]. Let $q$ be $2 p-2$ if $p$ is an odd prime and 8 if $p=2$. Then there is a map

$$
v: \Sigma^{q} M(p) \rightarrow M(p)
$$

inducing an isomorphism in $K$-theory. Hence all iterates of this $v$ are nontrivial.

The stable composite

$$
S^{q} \rightarrow \Sigma^{q} M(p) \stackrel{v}{\longrightarrow} M(p) \rightarrow S^{1}
$$

is $\alpha_{1}$ (the first element of order $p$ in $\pi_{*}^{S}$ ) for $p$ odd and $8 \sigma$ (where $\sigma$ is the generator of $\pi_{7}^{S}$ ) for $p=2$. The first and third maps here are respectively the inclusion of the bottom cell in $M(p)$ and the pinch map from $M(p)$ to its top cell.

If $X$ is the sphere spectrum then $\pi_{*}(X ; M(p))$ is the set of stable maps from $M(p)$ to a sphere. By $S$-duality this is isomorphic to $\pi_{*}^{S}(M(p)$ ) (up to reindexing) with $v$ acting by composition on the right instead of the left.

More generally for a finite spectrum $Y$ we have a similar isomorphism

$$
\pi_{*}(X ; Y)=\pi_{*}(X \wedge D Y)
$$

where $D Y$ is the Spanier-Whitehead dual of $Y$, i.e. the complement of the finite complex $Y$ embedded in a suitable sphere. In most cases of interest (e.g. $Y=M(p)$ ), $Y$ is self-dual, i.e. $D Y$ is some suspension of $Y$.

For $p=2$ the $v$-torsion free quotient of

$$
\pi_{*}\left(S^{0} ; M(p)\right)
$$

was first determined in [M3]. The following odd primary analog was proved by Miller.

Theorem 3. [Mi]. For an odd prime $p, \pi^{S_{*}}(M(p))$ mod v-torsion is generated by two elements represented by the stable composites

$$
\begin{array}{cccccc}
S^{q} & \longrightarrow & \Sigma^{q} M(p) & \stackrel{v}{\longrightarrow} & M(p) & \text { and } \\
S^{q-1} & \stackrel{\alpha_{1}}{\longrightarrow} & S^{0} & \longrightarrow & M(p) .
\end{array}
$$

The result for $p=2$ is considerably more complicated. There are ten generators instead of two and the group is a module over $Z /(4)[v]$ instead of $Z /(2)[v]$. The details need not concern us here.

What does this tell us about the stable homotopy groups of spheres? There is a long exact sequence relating ordinary and $\bmod (p)$ stable homotopy groups. In it the mod 
$(p)$ groups in Miller's theorem correspond to the $p$-component of the image of the stable $J$-homomorphism, which we will now describe.

There is a homomorphism

$$
J: \pi_{k}(S O(n)) \rightarrow \pi_{n+k}\left(S^{n}\right)
$$

defined originally by Hopf and Whitehead. Here $S O(n)$ denotes the group of $n \times n$ orthogonal matrices with determinant one.

Letting $n$ go to $\infty$ gives a homomorphism

$$
J: \pi_{k}(S O) \rightarrow \pi_{k}^{S}\left(S^{0}\right)
$$

where $S O$ denotes the stable orthogonal group. Its homotopy groups were determined by Bott, who showed that

$$
\begin{aligned}
\pi_{k}(S O) & =Z \text { if } k \equiv 3 \bmod (4), \\
& =Z /(2) \text { if } k \equiv 0 \text { or } 1 \bmod (8) \text { and } \\
& =0 \text { otherwise. }
\end{aligned}
$$

Adams showed in [A2] that $J$ is monomorphic on the 2-torsion and that its image on each free summand is a cyclic group whose order is a certain arithmetic function of the dimension $k$. (Actually his work left an ambiguous factor of two which depended on the Adams conjecture.) In particular, the order of this cyclic group is divisible by an odd prime $p$ iff $k \equiv-1 \bmod (2 p-2)$.

\section{Some unstable results}

An unstable analog of Theorem 3 would describe the $v$-torsion free quotient of $\pi_{*}\left(S^{n} ; M(p)\right)$. Such a result for $n$ odd and $p=2$ was obtained in [M2]. For technical reasons it was necessary to replace $M(2)$ by the complex $Y=M(2) \wedge C P^{2}$ in order to get the cleanest possible description of the actual groups, but this should be regarded as a minor detail.

Recall that Serre's theorem gave a map to an infinite loop space, namely $K(Z, n)$, which induced an isomorphism modulo $p$-torsion. Ideally one would like to have a similar map to an infinite loop space inducing an isomorphism in mod $(p)$ homotopy modulo $v$-torsion, where $v$ is as in Adams' lemma above. The best we can do is the following.

Theorem 4. $[\mathrm{M} 2]$. Let $n=2 m+1$ and $p=2$. There is a map

$$
f: \Omega_{0}^{2 m+1} S^{2 m+1} \rightarrow \Omega^{\infty} R P^{2 m} \wedge J
$$

inducing an isomorphism in $\pi_{*}(; M(2))$ modulo $v$-torsion. Here the source is the degree 0 component of the indicated

loop space and the target is the 0th space in the $\Omega$-spectrum $R P^{2 m} \wedge J$ where $R P^{2 m}$ is $2 m$-dimensional real projective space and $J$ is a certain spectrum which will be described below.

This result is useful because the homotopy groups of the target can be computed explicitly. For example the mod (2) homotopy of $J$ is $v$-torsion free. This fact strengthens the analogy with Serre's theorem, which gave a map from $S^{2 m+1}$ to an infinite loop space with torsion free homotopy. 
The theorem does not assert that the map induces a surjection in homotopy or in mod (2) homotopy, but that the mod (2) kernel is precisely the $v$-torsion subgroup and that the cokernel is all $v$-torsion. In [M2] the cokernel is described up to an ambiguity related to the Kervaire invariant problem.

It is quite likely that there is an analogous result for every odd prime with $R P^{2 m}$ replaced by the $2 m(p-1)$-skeleton of $B \Sigma_{p}$, the classifying space for the symmetric group on $p$ letters.

Conjecture 5. The map $f$ in the theorem above induces an isomorphism in $K$-theory.

The $K$-theory of the target has been computed by Miller and Snaith, but we do not know how to compute the $K$-theory of the source.

Now we will describe the spectrum $J$. It is so named because its homotopy is nearly identical to the image of the $J$-homomorphism. There is a different version of this spectrum for each prime $p$. We will use the same notation for each. We will describe the odd primary version first.

Let $b u$ be the spectrum for connective complex $K$-theory. This spectrum can be built out of the various connected covers of $B U$, the classifying space for stable complex vector bundles. $J$ is the fiber of a certain map from $b u_{(p)}$ (the localization of $b u$ at the prime $p$ ) to $\Sigma^{q} b u_{(p)}$. This map induces an isomorphism in homotopy in dimensions not divisible by $2 p-2$. The homotopy of $J$ in positive dimensions is isomorphic to the $p$-component of the image of the stable $J$-homomorphism.

For $p=2$ the definition of $J$ is slightly different. It is the fiber of a map from $b o_{(2)}$ to $\Sigma^{4} b s p_{(2)}$. Here bo and $b s p$ are the spectra obtained from the $(8 k-1)$-connected covers of the spaces $B O$ and $B S p$, the classifying spaces for stable real and symplectic vector bundles respectively. Bott periodicity tells us that $\Omega^{4} B S p=Z \times B O$ and $\Omega^{4} B O=Z \times B S p$.

The homotopy of $J$ in positive dimensions is not isomorphic to the image of $J$. The latter maps monomorphically to the former, leaving a cokernel of $Z /(2)$ in dimensions congruent to 0 and $1 \bmod (8)$. These groups correspond to the homotopy elements $\mu_{8 k}$ and $\mu_{8 k+1}$ constructed in [A2].

For each prime $p$ the stable map

$$
S^{0}(p) \rightarrow J
$$

induces an isomorphism in $K$-theory, a surjection in homotopy and an isomorphisn in $v^{-1} \pi_{*}(; M(p))$.

\section{Enter $B P$-theory}

Recall that our basic setup is the following. We have a finite complex $Y$ and a map $v$ to $Y$ from some suspension of $Y$ such that all iterates of $v$ are nontrivial. Then we try to compute $v^{-1} \pi_{*}(X ; Y)$ for our favorite space $X$.

We have seen three examples of reslts of this sort, namely Theorems 1, 3 and 4 . In Theorems 1 and $4, X=S^{2 n+1}$. In the former case $Y=S^{1}$ and $v$ is the map of degree $p$. In Theorems 3 and $4, Y$ is a $\bmod (p)$ Moore space or spectrum and the self-map $v$ is that provided by Lemma 2 .

Before proceeding to the main theorem in each case one must prove that all of the iterates of $v$ are nontrivial. In the case of the degree $p$ map in Theorem 1 this can be done with ordinary integer homology. Since $H_{1}\left(S^{1}\right)=Z$, we know that the map of degree $p^{i}$ (the $i$ th iterate of $v$ ) is nonzero for all $i$. In other words all iterates of the maps are essential because the map induces an isomorphism in rational homology. 
The self-maps of Lemma 2 (used in Theorems 3 and 4) have nontrivial iterates because they induce isomorphisms in $K$-theory. $K$-theory could also be used to show that all iterates of the degree $p$ map are nontrivial. Ordinary homology would not suffice to prove that the maps of Lemma 2 are nontrivial.

$B P$-theory, like $K$-theory, is a generalized homology theory strong enough to show that these two self-maps are periodic, i.e. that all of their iterates are essential. We will explain how it applies in these two cases.

There is a different version of $B P$-theory for each prime $p$. (If one wants to work globally one can use $M U$-theory, which is the same thing as complex cobordism.) For any space $X, B P_{*}(X)$, the reduced $B P$-homology of $X$, is a graded module over the coefficient ring

$$
B P_{*}=Z_{(p)}\left[v_{1}, v_{2}, \cdots\right]
$$

where the dimension of $v_{i}$ is $2 p^{i}-2$ and $Z_{(p)}$ denotes the integers localized at the prime $p$.

The degree $p$ map on $S^{n}$ induces multiplication by $p$ in

$$
B P_{*}\left(S^{n}\right)=\Sigma^{n} B P_{*}
$$

For the self-maps in Lemma 2 we have $B P_{*}(M(p))=B P_{*} /(p)$ and $v$ induces multiplication by $v_{1}$ for $p$ odd and by $v_{1}^{4}$ for $p=2$. In the latter case there is no self map of $M(2)$ inducing multiplication by simply $v_{1}$. The 4-cell complex $Y=M(2) \wedge C P^{2}$ used in [M2] was chosen precisely becaue it does admit such a self-map.

Since the Adams self-maps of Lemma 2 induce multiplication by $v_{1}$ or some power of it, the homotopy elements derived from Theorems 3 and 4 are said to be $v_{1}$-periodic.

What other sorts of finite complexes with periodic self-maps might we have?

Several conjectures conerning this question were made in [R2] and a lot of progress has been made on them in the past three years. The most striking result, proved very recently by Devinatz, Hopkins and J. Smith, says that $B P$-theory is strong enough to detect any such self-map.

Nilpotence Theorem 6. [DHS]. Let $Y$ be a p-local finite complex and let

$$
v: \Sigma^{d} Y \rightarrow Y
$$

be a map inducing the trivial homomorphism is BP-homology. Then some iterate of $v$ is null homotopic.

Note that Nishida's theorem is a special case of this, since it is easy to show that any self-map of a sphere in a positive stem induces the trivial map in $B P$-homology.

It is known that for any finite complex $Y, B P_{*}(Y)$ is finitely presented as a $B P_{*}$-module. From this it follows easily that any periodic endomorphism of $B P_{*}(Y)$ has an iterate which is an idempotent composed with multiplication by some element $v$ in $B P_{*}$. For the sake of simplicity, assume that this idempotent is the identity. Then the internal properties of $B P$-theory tell us essentially that this $v$ must be a power of one of the polynomial generators $v_{n}$.

Moreover $n$ is uniquely determined by the module $B P_{*}(Y)$ in the following way. It is the smallest $n$ such that $v_{n}^{-1} B P_{*}(Y)$ is nonzero, i.e. such that $B P_{*}(Y)$ contains elements not annihilated by any power of $v_{n}$. It is known [JY] that if $v_{n}^{-1} B P_{*}(Y)$ is nonzero then so is $v_{m}^{-1} B P_{*}(Y)$ for all $m>n$. In this case we say that the finite complex $Y$ has type $n$. Until 
1983 it was not even known that such finite complexes exist for all $n$ [Mit]. A conjecture in $[R 2]$ that remains open says that every finite complex admits a periodic self-map.

$Y=M(p)$ has type 1 . If $Y$ has type $n$ then we can denote $v^{-1} \pi_{*}(X ; Y)$ by $v_{n}^{-1} \pi_{*}(X ; Y)$ and refer to the $v$-periodic and $v$-torsion elements as $v_{n}$-periodic and $v_{n}$-torsion elements respectively. We conjecture that the information $v^{-1} \pi_{*}(X ; Y)$ gives about $X$ depends only on $n$. More precisely,

Conjecture 7.. Let $Y$ and $Y^{\prime}$ be two p-local finite complexes of type $n$ and let $f$ be a map from $X_{1}$ to $X_{2}$. Then the following are equivalent:

(i) $f$ induces an isomorphism in $v_{n}^{-1} \pi_{*}(; Y)$,

(ii) $f$ induces an isomorphism in $v_{n}^{-1} \pi_{*}\left(; Y^{\prime}\right)$, and

(iii) $f$ induces an isomorphism in $v_{n}^{-1} B P_{*}()$.

\section{The chromatic filtration}

Now we will describe a way to codify all the information given by the groups $v_{n}^{-1} \pi_{*}(X ; Y)$ for all $n$ in the stable case, i.e. when $X$ and $Y$ are spectra.

We first need to introduce Bousfield localization [B]. Let $E_{*}$ be a generalized homology theory. A spectrum $Y$ is $E$-local if for every $E$-acyclic spectrum $X$ (i.e. a spectrum satisfying $\left.E_{*}(X)=0\right),[X, Y]=0$, i.e. there are no essential maps from $X$ to $Y$.

An E-localization of a spectrum $W$ is a map to an $E$-local spectrum $W^{\prime}$ which is an $E_{*}$-equivalence, i.e. a map inducing an isomorphism in $E$-homology. It is easy to show that if such a localization exists it is unique. If both $E$ and $W$ are connective then the $E$-localization of $W$ is simply an arithmetic localization or completion.

However, if either of them fail to be connective then the existence of the localization is far from obvious and its properties are hard to predict. For example, the $K$-theoretic localization of the sphere has nontrivial homotopy groups in arbitrarily large negative dimensions and its $\pi_{-2}$ is $Q / Z$. The localization of its $(-1)$-connected cover at an odd prime is the spectrum $J$ discussed earlier. More details can be found in [R2].

In [B] Bousfield proved that such localizations always exist. Let $L_{n} X$ denote the localization of $X$ with respect to $v_{n}^{-1} B P$. $L_{0} X$ is the rational localization of $X$; by convention $v_{0}=p . L_{1} X$ is the localization with respect to $p$-local $K$-theory. A $v_{n}^{-1} B P$-local spectrum is also $v_{n+1}^{-1} B P$ so there are natural maps

$$
L_{0} X \leftarrow L_{1} X \leftarrow \cdots
$$

We say that $X$ is harmonic if this inverse system converges to $X$. In [R2] it is shown that all $p$-local finite spectra are harmonic.

If $Y$ is a finite complex of type $n$ then one of the conjectures of [R2] implies that

$$
v_{n}^{-1} \pi_{*}(X ; Y)=\pi_{*}\left(L_{n} X ; Y\right)
$$

In other words $L_{n} X$ captures all the $v_{n}$-periodic information about $X$.

The inverse system above leads to a decreasing filtration of $\pi_{*}(X)$, defined by setting $F^{n} \pi_{*}(X)$ equal to the kernel of the homomorphism induced by the localization map from $X$ to $L_{n} X$, i.e. to the $v_{n}$-torsion. This is the chromatic filtration of $\pi_{*}(X)$. An algebraic analog of it, the chromatic spectral sequence, is studied extensively in [MRW] and in Chapter 5 of $[\mathrm{R} 1]$. 
Let $M_{n} X$ denote the fiber of the map from $L_{n} X$ to $L_{n-1} X$. Its homotopy is all $v_{n-1^{-}}$ torsion and all $v_{n}$-periodic. We know [R3] how to compute $B P_{*}\left(M_{n} X\right)$. Its AdamsNovikov spectral sequence is conjectured but unfortunately still not known to converge to $\pi_{*}\left(M_{n} X\right)$. It $E_{2}$-term is closely rrelated to the continuous mod $(p)$ cohomology of a certain pro- $p$-group with interesting arithmetic properties.

This connection was discovered over a decade ago by Morava [Mo]. Again we refer the reader to [MRW] or [R1] for more details. It means that this $E_{2}$-term is in some sense finitely computable, which is certainly not the case for most $E_{2}$-terms associated with finite complexes. For $n=1$ and $X=S^{0}$ this computation is done in complete detail in [MRW] and in Chapter 5 of [R1].

Admittedly there are infinitely many values of $n$ required for a complete understanding of the homotopy groups of spheres, but each of these finite calculations yields an infinite amount of information.

With Theorem 4 and related stable results we have a complete picture of the $v_{1}$-periodic homotopy groups (both stable and unstable) of spheres, at least for $p=2$.

We only have partial results for the $v_{2}$-periodic picture, which is considerably more complicated. There is no known $v_{2}$-periodic analog of either the $J$-homomorphism or the spectrum $J$.

It is also clear that things are simpler for larger primes. For $p>3$ let $V(1)$ denote the cofiber of the Adams self-map of Lemma 2. It is the simplest complex of type 2. The conjectures of [R2] imply that

$$
v_{2}^{-1} \pi_{*}\left(S^{0} ; V(1)\right)
$$

has precisely 12 generators. The image of $\pi_{*}\left(S^{0} ; V(1)\right)$ in this group is still unknown.

\section{The EHP sequence}

The EHP sequence is the fundamental tool for understanding unstable homotopy groups of spheres. A more thorough exposition can be found in the last section of Chapter 1 of [R1]. For simplicity we limit our remarks to the prime 2. Most of what is said below has an analog at any odd prime, but the statements are more complicated.

There are fibrations

$$
S^{n} \stackrel{E}{\longrightarrow} \Omega S^{n+1} \stackrel{H}{\longrightarrow} \Omega S^{2 n+1}
$$

for all positive $n$. The map $E$ stands for suspension (Einhängung) and $H$ for Hopf invariant. The associated long exact sequence of homotopy groups is the EHP sequence.

Collectively (for all $n$ ) these long exact sequences constitute an exact couple which leads to what is called the EHP spectral sequence. Many classical results about unstable homotopy groups can be translated into statements about this spectral sequence. Such results include the Freudenthal Suspension Theorem (see Section 1 above) and the Adams Vector Field Theorem of [A1]. Theorem 4 has implications concerning certain patterns in the EHP spectral sequence, specifically the behavior of elements whose Hopf invariant lies in the image of $J$ (hence the title of [M2]).

The following result of Snaith, enhanced by an observation of Kuhn, relates unstable groups appearing in the EHP spectral sequence to stable groups.

Theorem 8. $[\mathrm{Sn}][\mathrm{K}]$. There are maps

$$
f: \Omega^{n+1} S^{n+1} \rightarrow Q R P^{n}
$$


(where $Q X$ denotes $\Omega^{\infty} \Sigma^{\infty} X$ ) such that the following diagram commutes up to homotopy.

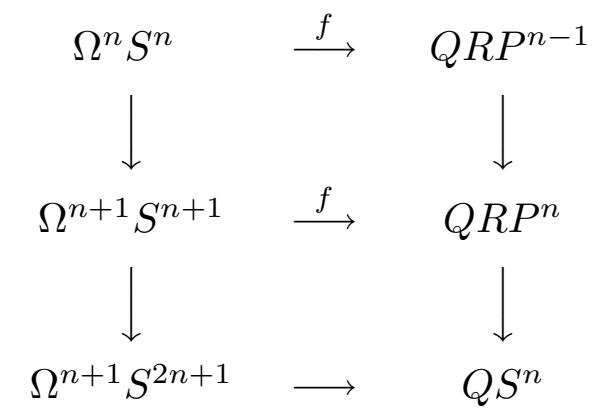

The vertical maps on the left are $\Omega^{n} E$ and $\Omega^{n} H$ while the ones on the right are the images under the functor $Q$ on the evident cofiber sequence.

Using this result one can easily produce a map from the EHP spectral sequence to the Atiyah-Hirzebruch spectral sequence for the stable homotopy of $R P^{\infty}$, in which all groups in sight are stable.

There are versions of the EHP spectral sequence converging to the stable homotopy groups of spheres and to the unstable homotopy groups of any given sphere. The $E_{1^{-}}$ term also consists of unstable homotopy groups, so it lends itself very well to inductive calculation. The input at each stem consists of the output from lower stems. In principle all one needs to start the inductive process is knowledge of the homotopy groups of $S^{1}$, which are easily determined.

The entire EHP apparatus can be adapted to the computation of $\pi_{*}\left(S^{n} ; Y\right)$. The following result shows that each element in a $v_{n}$-periodic family (with a finite number of exceptions for any given family) has a $v_{n}$-periodic Hopf invariant, and that all but finitely many elements in each such family originate on the same sphere. More precisely we have

Theorem 9. Let $Y$ be a finite complex of type $n$ with a self-map $v$ inducing multiplication by some power of $v_{n}$ in its BP-homology. If

$$
x \in \pi_{*}\left(S^{m} ; Y\right)
$$

is $v$-periodic, then there is a $k \leq m$ such that some $v$-multiple of $x$ is the iterated suspension of

$$
x^{\prime} \in \pi_{*}\left(S^{k} ; Y\right)
$$

and the Hopf invariant

$$
H\left(x^{\prime}\right) \in \pi_{*}\left(S^{2 k-1} ; Y\right)
$$

is v-periodic. Hence all higher $v$-multiples of $x$ are born on $S^{k}$.

Proof. Suppose $H\left(x^{\prime}\right)$ is not $v$-periodic. Then by definition some power of $v$ annihilates it. Thus the corresponding $v$-multiple of $x^{\prime}$ desuspends further since the Hopf invariant is the obstruction to desuspension. This process must stop after a finite number of steps because we cannot desuspend below the 1-sphere. Thus some $v$-multiple of $x$ desuspends to an element with a $v$-periodic Hopf invariant as claimed, and all higher $v$-multiples of $x$ are born on $S^{k}$.

Very early work with the EHP sequence showed that the $p$-torsion subgroups of the homotopy of each finite sphere has a bounded exponent. The best possible result of 
this sort for odd primes was proved by Cohen-Moore-Neisendorfer [CMN]. Experimental evidence suggests that there should be similar bounds on the $v_{n}$-torsion in suitable groups for finite spheres.

\section{James periodicity and the root invariant}

Theorem 8 shows a connection between the EHP sequence and the homotopy groups of stunted projective spaces. For $m \leq n$ let $R P_{m}^{n}$ denote $R P^{n} / R P^{m-1}$, i.e. the stunted projective space with bottom cell in dimension $m$ and top cell in dimension $n$. There is an equivalence

$$
\Sigma^{K} R P_{m}^{n} \rightarrow R P_{m+K}^{n+K}
$$

if $K$ is a sufficiently large (depending on $n-m$ ) power of 2. This leads to a certain regularity in the EHP sequence called James periodicity. It is not related to the $v_{n^{-}}$ periodicity discussed elsewhere in this paper.

It is possible to define spectra $R P_{m}^{n}$ and $R P_{m}^{\infty}$ (denoted simply by $R P_{m}$ ) for arbitrary (e.g. negative) integers $m$. There are maps

$$
R P_{m} \leftarrow R P_{m-1} \leftarrow \cdots
$$

obtained by pinching out the bottom cell in each case.

Theorem 10. Lin [L]. The homotopy inverse limit of the $R P_{m}$ (denoted by $R P_{-\infty}$ ) is homotopy equivalent to the 2-adic completion of $S^{-1}$ in such a way that the composite map

$$
S^{-1} \rightarrow R P_{-\infty} \rightarrow R P_{-1}
$$

is homotopic to the inclusion of the bottom cell.

Theorem 11. Kahn-Priddy $[\mathrm{KP}]$. The inclusion of the bottom cell in $R P_{-1}$ induces the trivial homomorphism in all homotopy groups except $\pi_{-1}$, in which the image is $Z /(2)$.

Theorem 10 means that the Atiyah-Hirzebruch spectral sequence for the stable homotopy of $R P_{-\infty}$ must converge to that of $S^{-1}$. This has certain implications for the EHP sequence. In particular it allows us to define a mutation of the Hopf invariant which we call the root invariant.

Suppose $\alpha \in \pi_{n}^{S}$ is represented by a map

$$
S^{n-1} \rightarrow S^{-1}
$$

If $n$ is positive or $n=0$ and $\alpha$ is divisible by two then the composite

$$
S^{n-1} \rightarrow S^{-1} \rightarrow R P_{-1}
$$

is null homotopic by Theorem 11 .

On the other hand Theorem 10 guarantees that for some $m$ the composite

$$
S^{n-1} \rightarrow S^{-1} \rightarrow R P_{-m}
$$

is essential. 
Taking the smallest such $m$ we get a factorization

$$
S^{n-1} \rightarrow S^{-m} \rightarrow R P_{-m}
$$

defined modulo a certain indeterminacy.

The resulting coset in

$$
\pi_{n-1}\left(S^{-m}\right)=\pi_{m+n-1}\left(S^{0}\right)
$$

is the root invariant $R(\alpha)$. Notice that we started in the $n$-stem and ended up in the $(n+m-1)$-stem, where the number $m$ depends on $\alpha$ as well as on $n$. It is known that in general $m \geq 2 n+1$. Many root invariants have been calculated. For example we have

$$
\begin{aligned}
\operatorname{dim} R\left(2^{i}\right) & =2 i-1 \text { if } i \equiv 0 \text { or } 1 \bmod (4) \\
& =2 i-2 \text { if } i \equiv 2 \bmod (4) \\
& =2 i-3 \text { if } i \equiv 3 \bmod (4)
\end{aligned}
$$

and $R\left(2^{i}\right)$ contains the element of order two in the image of $J$ if $i \equiv 0$ or $3 \bmod (4)$. It is conjectured that $R\left(\theta_{j}\right)$ contains $\theta_{j+1}$ if both exist, where $\theta_{j}$ is the Kervaire invariant element.

The definition of the root invariant can be adapted to homotopy with coefficients in $Y$. Experimental evidence suggests that the root invariant of a $v_{n}$-periodic element is $v_{n+1}$-periodic. More precisely we have

Conjecture 12. If $Y$ is of type $n$ and has a $v_{n}$-periodic self-map $v$

$$
\alpha \in \pi_{*}\left(S^{-1} ; Y\right)
$$

is $v$-periodic then the coset $R(\alpha)$ consists entirely of $v_{n}$-torsion elements. Let

$$
w: \Sigma^{d} Y \rightarrow Y
$$

be a power of $v$ which annihilates every element in $R(\alpha)$ and let $Z$ be its cofiber. Thus $Z$ has type $n+1$ and each element in $R(\alpha)$ extends to a map from $Z$ to a suitable sphere. At least one of these maps is $v_{n+1}$-periodic.

\section{REFERENCES}

[A1] J.F. Adams, Vector fields on spheres, Ann. of Math., 75 (1962), 603-632.

[A2] J.F. Adams, The groups $J(X)$, IV, Topology, 5 (1966), 21-71.

[B] A.K. Bousfield, Localization of spaces with respect to homology, Topology, 18 (1979), $257-281$.

[CMN] F.R. Cohen, J.C. Moore and J. Neisendorfer, The double suspension and exponents of the homotopy groups of spheres, Ann. of Math., 110 (1979), 549-565.

[DHS] E. Devinatz, M. Hopkins and J. Smith, Nilpotence in stable homotopy theory, to appear.

[JY] D.C. Johnson and Z. Yosimura, Torsion in Brown-Peterson homology and Hurewicz homomorphisms, Osaka J. Math., 17 (1980), 117-136.

[KP] D.S. Kahn and S.B. Priddy, The transfer and stable homotopy, Math. Proc. Camb. Phil. Soc., 83 (1978), 103-111. 
[K] N. Kuhn, The geometry of the James-Hopf maps, Pac. J. Math., 102 (1982), 397-412.

[L] W.H. Lin, On conjectures of Mahowald, Segal and Sullivan, Math. Proc. Camb. Phil. Soc., 87 (1980), 449-458.

[M1] M.E. Mahowald, The metastable homotopy of $S^{n}$, Memoirs Amer. Math. Soc. No. 72, 1967.

[M2] M.E. Mahowald, The image of $J$ in the EHP sequence, Ann. of Math., 116 (1982), 65-112.

[M3] M.E. Mahowald, bo-resolutions, Pacific J. Math., 192 (1981), 365-383.

[Mi] H.R. Miller, On relations between Adams spectral sequences, with an application to the stable homotopy of a Moore spaces, J. Pure Appl. Alg., 20 (1981), 287-312.

[MRW] H.R. Miller, D.C. Ravenel and W.S. Wilson, Periodic phenomena in the Adams-Novikov spectral sequence, Ann. of Math., 106 (1977), 469-516.

[Mit] S.A. Mitchell, Finite complexes with $A_{n}$-free cohomology, to appear in Topology.

[Mo] J. Morava, Structure theorems for cobordism comodules, to appear somewhere.

[N] G. Nishida, The nilpotency of elements in the stable homotopy groups of spheres, $J$. Math. Soc. Japan, 25 (1973), 707-732.

[R1] D.C. Ravenel, Complex cobordism and stable homotopy groups of spheres, Academic Press, New York, 1986.

[R2] D.C. Ravenel, Localization with respect to certain periodic homology theories, Amer. J. Math., 106 (1984), 351-414.

[R3] D.C. Ravenel, The geometric realization of the chromatic resolution, Proc. of Conference in Honor of J.C. Moore, to appear.

[S] J.-P. Serre, Groupes d'homotopie et classes de groupes abelien, Ann. of Math., 58 (1953), 258-294.

[Sn] V.P. Snaith, Stable decomposition of $\Omega^{n} \Sigma^{n} X$, J. London Math. Soc., 7 (1974), 577-583.

Northwestern University, Evanston, IL 60201

University of Washington, Seattle, WA 98195

Both authors partially supported by the N.S.F. 